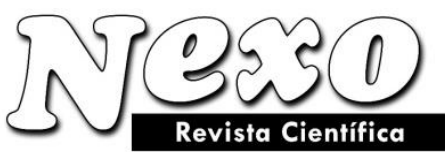

Vol. 34, No. 02, pp. 572-582/Junio 2021
ISSN-E 1995-9516

Universidad Nacional de Ingeniería COPYRIGHT @ (UNI). TODOS LOS DERECHOS RESERVADOS http://revistas.uni.edu.ni/index.php/Nexo https://doi.org/10.5377/nexo.v34i02.11542

\title{
Caracterización de la macrofauna edáfica en diferentes sistemas agroforestales, en el Municipio de San Ramón, Departamento de Matagalpa, Nicaragua
}

\section{Characterization of the edaphic macrofauna in different agroforestry systems, in the Municipality of San Ramón, Department of Matagalpa, Nicaragua}

\author{
Conrado R. Quiroz Medina*, Juan D. Castellón, Noelia E. Cea Navas, Mirna S. Ortiz, Carlos A. Zuniga- \\ Gonzalez \\ Universidad Nacional Autónoma de Nicaragua, León. Escuela de Ciencias Agrarias y \\ Veterinarias. Departamento de Agroecología. \\ conrado.quiroz@ev.unanleon.edu.ni
}

(recibido/received: 17-febrero-2021; aceptado/accepted: 10-mayo-2021)

\section{RESUMEN}

El presente estudio de focalizo en caracterizar la macrofauna edáfica en tres diferentes sistemas agroforestales: CMIEA: Café, musáceas, Inga, Erithryna y árboles, CIE: Café, Inga, Erithryna y árboles y CA: Café y árboles en época seca y época lluviosa. Cada sistema tenía $900 \mathrm{~m}^{2}$, presentaban aproximadamente 6 especies arbóreas por sistema. Se tomaron 32 monolitos por época, y este se dividió en cuatros estratos (hojarasca, 0 a $10 \mathrm{~cm}, 10$ a $20 \mathrm{~cm}$ y 20 a $30 \mathrm{~cm}$ ) con el método recomendado por el Tropical Soil Biology and Fertility Programme. La macrofauna se conservó en alcohol al 70\%, exceptuando las lombrices que fueron preservadas con formalina al $4 \%$ y se trasladaron al Laboratorio de Entomología del Departamento de Agroecología de la UNAN-León para su respectiva edificación. Los resultados demuestran que los valores mayores de la abundancia se presentaron en la época lluviosa (391 individuos) y en la época seca (324 individuos). La mayor abundancia se encontró en el sistema CMIEA (162 individuos) en la época lluviosa y mínima en el sistema CA (91 individuos) en la época seca. El índice de diversidad de Shannon fue más alto en el sistema CA (2.73) en la época seca y mínimo en el sistema CIE (1.44). Los arreglos de los sistemas agroforestales no influyen en la abundancia de la macrofauna, sin embargo, favorece la presencia de especie de Philoscidae y lombrices de suelos orden Haplotaxida.

Palabras claves: Agroforestal; Monolito; Estrato

\begin{abstract}
The present study was focused on order to characterize the edaphic macrofauna in three different agroforestry systems: CMIEA: Coffee, musaceae, Inga, Erithryna and trees, CIE: Coffee, Inga, Erithryna and trees and CA: Coffee and trees in dry season and rainy season. Each system had $900 \mathrm{~m}^{2}$, they had approximately 6 tree species per system. 32 monoliths were taken per season, and this was divided into four strata (litter, 0 to $10 \mathrm{~cm}, 10$ to $20 \mathrm{~cm}$ and 20 to $30 \mathrm{~cm}$ ) with the method recommended by the Tropical Soil Biology and Fertility Program. The macrofauna was preserved in $70 \%$ alcohol, except for the worms that were preserved with $4 \%$ formalin and were transferred to the Entomology Laboratory of the Department of Agroecology of UNAN-León for their respective construction. The results show the highest values of
\end{abstract}


abundance occurred in the rainy season (391 individuals) and in the dry season (324 individuals). The highest abundance was found in the CMIEA system (162 individuals) in the rainy season and minimum in the CA system (91 individuals) in the dry season. The Shannon diversity index was highest in the CA system (2.73) in the dry season and lowest in the CIE system (1.44). The arrangements of the agroforestry systems do not influence the abundance of the macrofauna, however, it favors the presence of Philoscia species and soil worms order Haplotaxida.

Keywords: Agroforestry; Monolith; Stratum

\section{INTRODUCCIÓN}

Debido a las preocupaciones por la degradación de los suelos y la necesidad de una gestión sostenible de este recurso, se han venido sumando en la comunidad científica una renovada atención sobre la evaluación de bioindicadores de calidad de suelo, Lok, (2005); Chaves et al. (2018).

Estudios realizados han sugerido que la macrofauna puede ser considerada como indicador temprano y sensible a los efectos del riesgo y manejo de los suelos (Govaerts, et al. (2006); García, et al. (2014)).

Según García, (2014) en el trópico, la macrofauna es una de las más representativa dentro del suelo; esta contiene invertebrados con un diámetro mayor de $2 \mathrm{~mm}$ y fácilmente visible en la superficie y dentro del suelo. Lavelle et al. (2006) menciona que estos organismos desempeñan un papel importante de alterar el ambiente superficial y edáfico en que se desarrollan las plantas.

Al manejar adecuadamente la diversidad vegetal en un agroecosistema, se aseguran mayor diversidad y abundancia de organismo vivos en el suelo, dando así sostenibilidad del mismo, Primavesi, (1984). La pérdida de materia orgánica constituye uno de los principales factores que influyen en los bajos valores de la densidad de la macrofauna del suelo, Coral y Bonilla, (1998). El objetivo de este trabajo es estudiar el comportamiento de la macrofauna edáfica en diferentes sistemas agroforestales en periodo seco y lluvioso.

\section{Sitio y área de estudio}

\section{MATERIALES Y METODOS}

El estudio fue realizado en el Municipio de San Ramón, departamento de Matagalpa a $12^{\circ} 57^{\prime} 49.50^{\prime}$ N $\mathrm{N}$

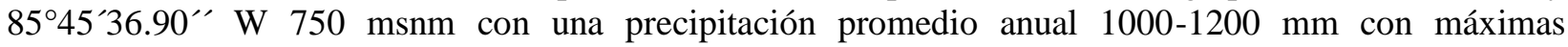
precipitaciones mayo, agosto, septiembre y octubre, temperatura promedio de $24^{\circ} \mathrm{C}$ y humedad relativa de $87 \%$. La zona presenta una pendiente del $34 \%$ con un suelo de franco arcilloso a arenoso. La zona de vida es un bosque tropical húmedo premontano.

\section{Tipo de estudio}

El tipo de estudio que se realizó fue descriptivo del tipo transeccional, en este tipo de investigación no se manipula las variables, se observan los fenómenos a como se dan en su contexto natural. Los diseños transeccionales se recolectan los datos en un solo momento, en un tiempo único.

\section{Descripción de los sitios estudiados}

Las muestras de la macrofauna de suelo fueron recolectadas en tres sistemas agroforestales (Tabla 1) en un área experimental de $900 \mathrm{~m}^{2}$ por sistema. En los tres sistemas agroforestales contenía cultivo de café a una distancia de siembra de $2 \mathrm{~m}$ entre surco por $1.5 \mathrm{~m}$ entre planta.

En el establecimiento de los sistemas se conservó las especies arbóreas, encontrando 6 especies diferentes por sistema, realizando dos podas por año. 
Tabla 1. Descripción del arreglo de los sistemas agroforestales

\begin{tabular}{|c|c|}
\hline $\begin{array}{l}\text { Código del } \\
\text { sitio }\end{array}$ & Descripción \\
\hline CMIEA & $\begin{array}{l}\text { Sistema agroforestal compuesto de café (Coffea arabica), musácea a una distancia } \\
\text { de siembra de } 5 \mathrm{~m} \text { entre planta por } 4 \mathrm{~m} \text { entre surco, especies arbustiva Inga a } 2.5 \mathrm{~m} \\
\text { entre planta y } 4 \mathrm{~m} \text { entre surco y Erithryna a } 5 \mathrm{~m} \text { entre planta y } 4 \mathrm{~m} \text { entre surco y } \\
\text { árboles disperso. }\end{array}$ \\
\hline CIE & $\begin{array}{l}\text { Sistema agroforestal compuesto de café (Coffea arabica) y especies arbustiva de } \\
\text { Eritrhyna e Inga a una distancia de } 2.5 \mathrm{~m} \text { entre planta y } 4 \mathrm{~m} \text { entre surco en ambas } \\
\text { especies. }\end{array}$ \\
\hline $\mathrm{CA}$ & En este sistema solo se estableció el cultivo de café (Coffea arabica). \\
\hline
\end{tabular}

Fuente: Elaboración propia

\section{Descripción del método de muestreo}

Para la recolecta de la macrofauna edáfica se utilizó la metodología propuesta por Tropical Soil Biology and Fertility, Anderson e Ingran, (1993).

Cada monolito tenía la dimensión de 25 × 25 x $30 \mathrm{~cm}$. La recolecta se realizó manualmente in situ, hasta los $30 \mathrm{~cm}$ de profundidad explorando los estratos de suelo: hojarasca, $0-10 \mathrm{~cm}, 10-20 \mathrm{~cm}$ y $20-30 \mathrm{~cm}$. El muestreo se realizó en abril (época seca) y en agosto (época lluviosa) del 2015, en cada sistema agroforestal se tomó 12 monolito en dos transectos en forma de X a 7m cada monolito según, Moreira et al. (2012).

La determinación taxonómica del material se realizó hasta el nivel de género, basados en los trabajos realizados con la taxonomía (Adrews y Caballero, (1990); King, y Saunders, (1984); Maas, J.L (1998); Coto-Alfaro, (1998); Serna y Vergara, (2001), se realizaron comparaciones de los especímenes con las imágenes publicadas de la página de BudGuide ${ }^{1}$.

Los especímenes encontrados se organizaron en viales rotulados, las lombrices de tierra se fijaron con formalina al $4 \%$ y el resto de los invertebrados con alcohol al $70 \%$.

\section{Análisis estadístico}

Para determinar la diferencia significativa entre los sistemas agroforestales se realizó la prueba no paramétrica de Kruskal-Wali. El paquete estadístico utilizado fue SPSS vr 23 para Windows. Para todo el análisis se utilizó un nivel de significancia de 0.05 .

\section{RESULTADOS Y DISCUSION}

\section{Composición y riqueza taxonómica}

Durante la investigación se extrajo 32 monolitos por época y se recolectaron 715 individuos en las dos épocas. El número de individuos recolectados fue más alto en la época lluviosa (391 individuos) en comparación con los recolectados en la época seca (324 individuos). Para el sistema agroforestal CNIEA en la época lluviosa se encontró 163 individuos mientras que en el sistema agroforestal CIE se encontró 111 individuos y en el sistema agroforestal CA 117 individuos. Para la época seca en el sistema CNIEA se obtuvieron 129 individuos, en el sistema CIE 104 individuos y en el sistema CA 91 individuos (Figura 1).

\footnotetext{
${ }^{1}$ disponible en: https://bugguide.net/node/view/15740 visitado en febrero 2021.
} 


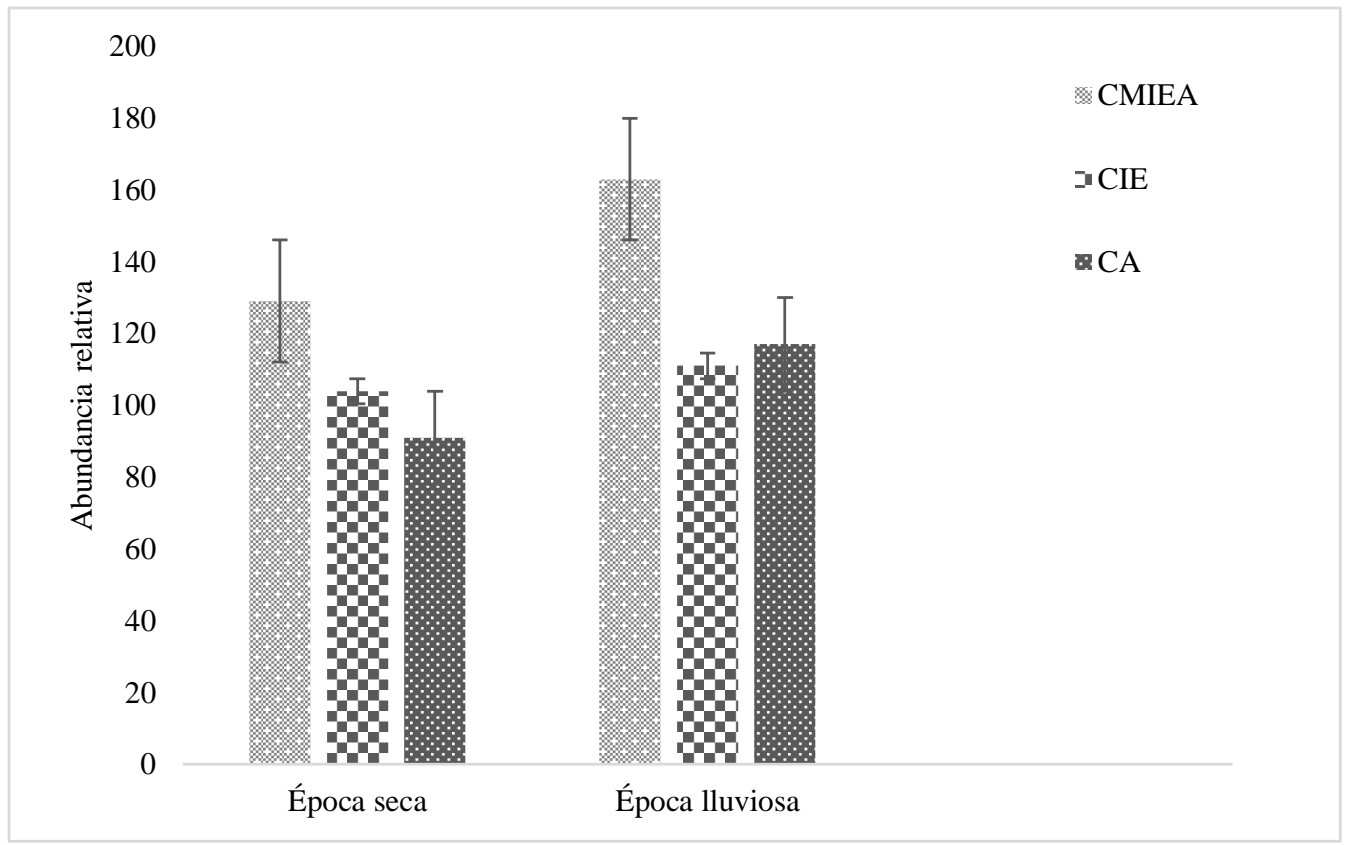

Figura 1. Abundancia relativa de macrofauna en diferentes sistemas agroforestal en época seca y lluviosa. CMIEA= Café, musáceas, Inga, Erythrina y árboles; CIEA=Café, Inga y Erythrina y árboles; CA= Café y Árboles.

La composición taxonómica del estudio para la época lluviosa se encontraron 26 familias mientras que en la época seca 30 familias. Es de señalar que los organismos más representados en abundancia fueron las lombrices pertenecientes al orden Haplotaxida con 208 individuos en época de invierno y 108 individuos en época seca, la familia Philosciidae con 92 individuos en época lluviosa y 24 individuos en época seca y la familia Formicidae con 25 individuos en época lluviosa y 23 individuos en época seca (Ver apéndice Tabla 3).

La mayor cantidad de macrofauna se encontró en el estrato $0-10 \mathrm{~cm}$, para el sistema agroforestal CMIEA con 80 individuos, seguido por el sistema CA con 72 individuos, mientras que en el sistema CIE 69 individuos en la época de invierno (Figura 2). Para la época de verano de igual manera la mayor cantidad de invertebrados fueron encontrados en el estrato de $0-10 \mathrm{~cm}$ predominando el sistema agroforestal CIE con 62 individuos, seguido por el sistema CMIEA con 57 individuos, mientras que el sistema CA se encontró 47 individuos (Figura 3). Rendon et, al. (2011) demuestro que el mayor número de macroinvertebrados fue encontrado en el estrato de $0-10 \mathrm{~cm}$ con un $87 \%$ de los individuos recolectados. Primavesi (1984) asegura que a medida que aumenta la profundidad del suelo disminuye la materia orgánica y el oxígeno. Pardo et, al. (2006) menciona que la mayor cantidad de invertebrados en cafetal con sombrío se encuentra en el estrato de $0-10 \mathrm{~cm}$, esto es atribuido por la biomasa vegetal presente en los primeros $10 \mathrm{~cm}$ de profundidad. 




Figura 2. Abundancia relativa de macrofauna por estrato en los diferentes sistemas agroforestal en período invierno. CMIEA= Café, musáceas, Inga, Erythrina y árboles; CIEA=Café, Inga y Erythrina y árboles; CA= Café y Árboles.

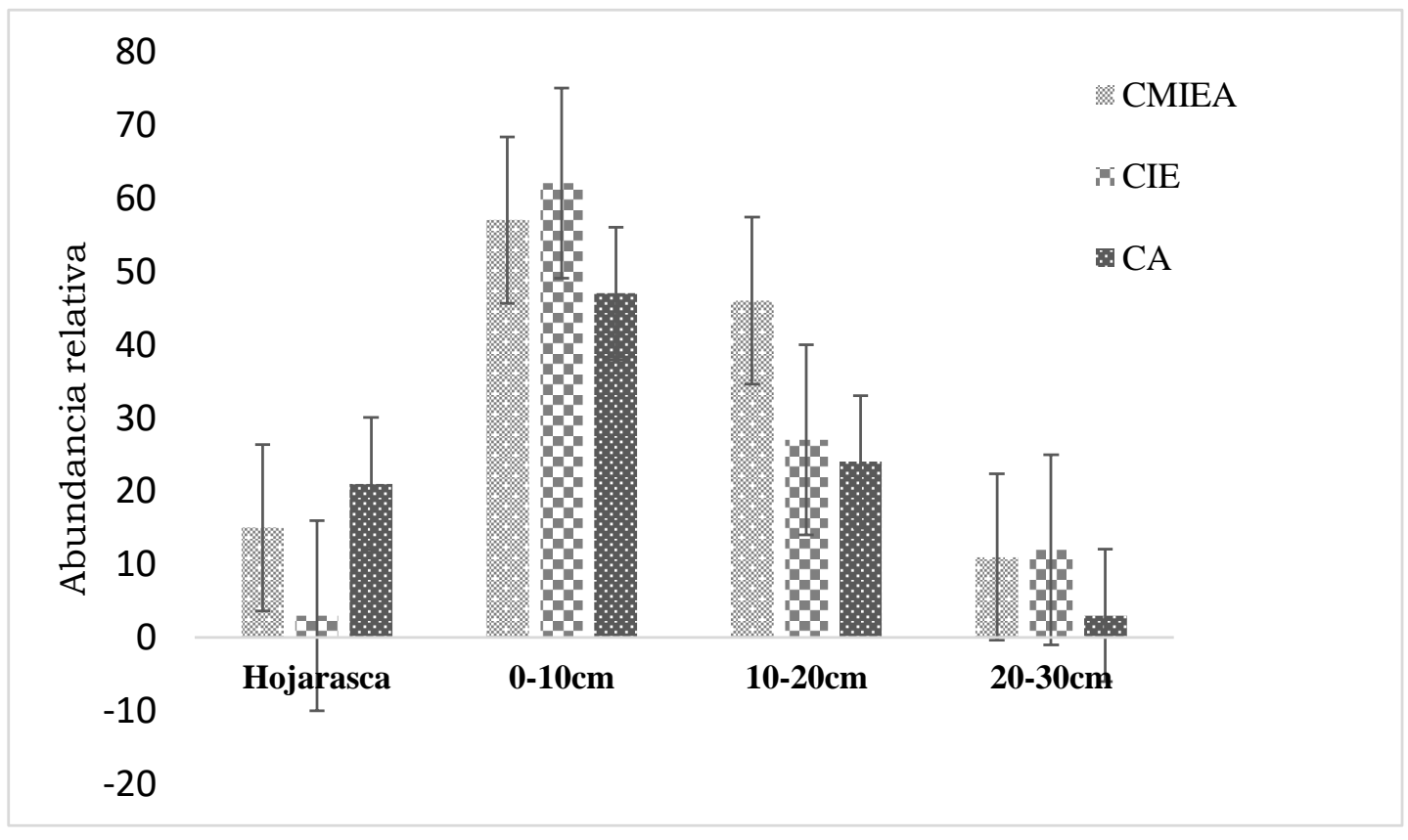

Figura 3. Abundancia relativa de macrofauna por estrato en los diferentes sistemas agroforestal en período verano. CMIEA= Café, musáceas, Inga, Erythrina y árboles; CIEA=Café, Inga y Erythrina y árboles; CA= Café y Árboles.

El índice de la diversidad de Shannon fue más alto en el sistema agroforestal CA (2.73) en la época seca, seguido por el sistema CMIEA (2.62) en la época lluviosa. Los sistemas agroforestales que presentaron 
índice alto en las dos épocas del año fueron el CA y CMIEA (Tabla 2). El índice de Shannon aumenta cuando el número de individuos y la riqueza son uniforme, sin embargo, el sistema CMIEA presentó mayor abundancia en las dos épocas del año, pero presento menor índice en comparación al sistema CA, debido a que la abundancia no está distribuida uniformemente con respecto al número de especies encontradas. Blair et., al (1996) menciona, que los invertebrados del suelo son considerados como indicadores de calidad del suelo debido a que su diversidad, su número y sus funciones son sensibles a las actividades antrópicas haciendo disminuir su diversidad y condicionando el hábitat a otros organismos.

\begin{tabular}{|c|c|c|}
\hline SistemaAgroforestal & $\begin{array}{l}\text { E. Lluviosa } \\
\text { índice de } \\
\text { shannon }\end{array}$ & $\begin{array}{l}\text { E. Seca } \\
\text { Indice de } \\
\text { shannon }\end{array}$ \\
\hline CMIEA & 2.62 & 2.34 \\
\hline CIE & 1.42 & 1.44 \\
\hline $\mathrm{CA}$ & 2.52 & 2.73 \\
\hline
\end{tabular}

De acuerdo con la abundancia relativa de los grupos funcionales de la macrofauna edáfica, en la época seca se observó que el grupo detritívoro predominó en los tres sistemas agroforestales con mayor abundancia en el sistema CMIEA con 97 individuos seguido por el sistema CIE con 91 individuos (Figura 4). De igual manera para la época lluviosa los detritívoros predominaron en los tres sistemas agroforestales resaltando en el sistema CMIEA con 117 individuos, seguido por el sistema CIE con 102 individuos (Figura 5), sobresaliendo las lombrices de suelo del orden haplotaxida y la familia Philosciidae en los tres sistemas agroforestales y en las dos épocas del año.

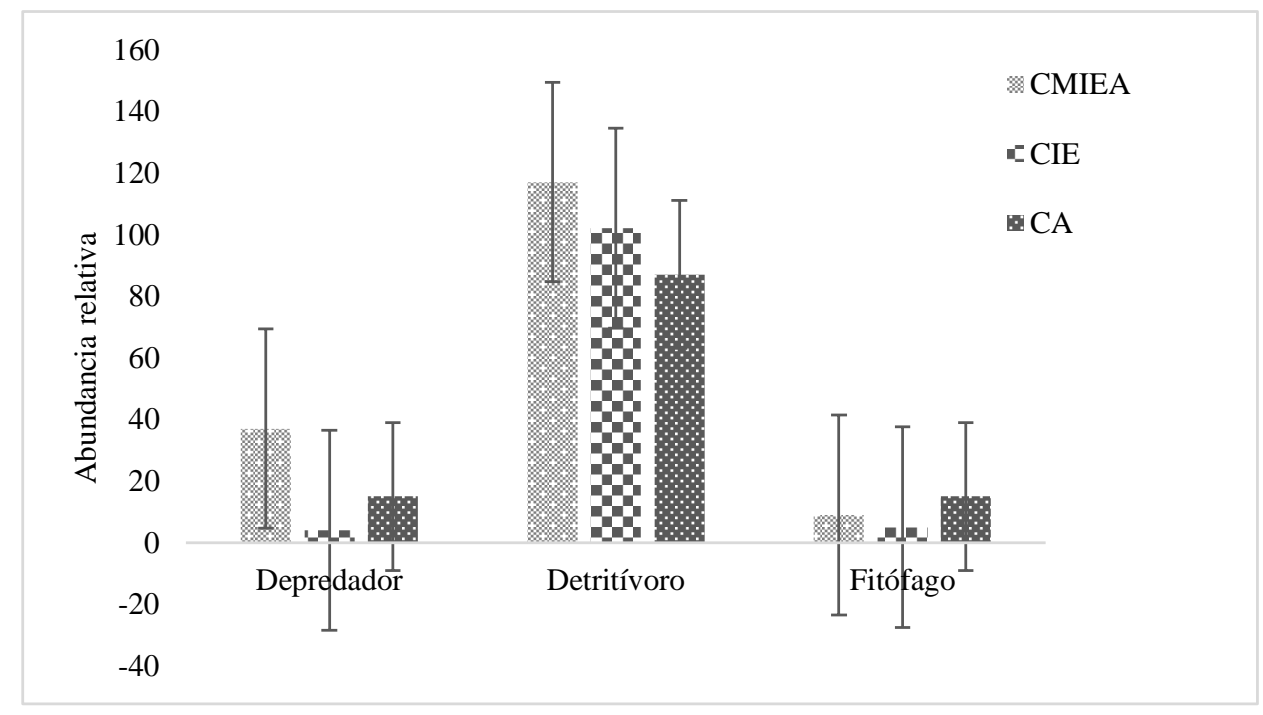

Figura 4. Grupo funcionales de la macrofauna edáfica en diferentes sistemas agroforestales en época seca.

CMIEA= Café, musáceas, Inga, Erythrina y árboles; CIEA=Café, Inga y Erythrina y árboles; CA= Café y Árboles. 


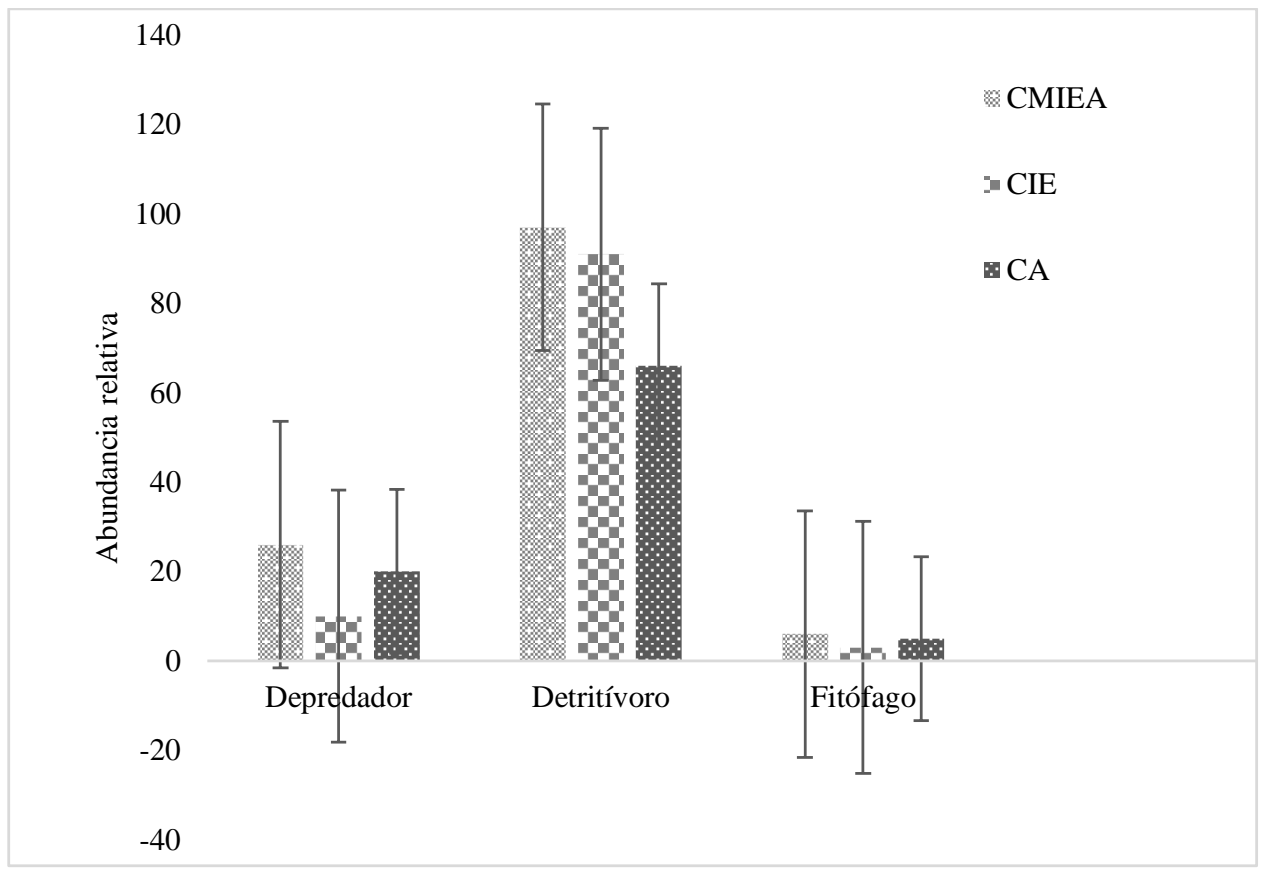

Figura 5. Grupo funcionales de la macrofauna edáfica en diferentes sistemas agroforestales en época lluviosa.

CMIEA= Café, musáceas, Inga, Erythrina y árboles; CIEA=Café, Inga y Erythrina y árboles; CA= Café y Árboles.

Sánchez, (2007); Sánchez et al., (2003), en experimentos de descomposición de la hojarasca por la macrofauna del suelo en ambientes con guinea y con leucaena más guinea, encontraron que el $77 \%$ de los organismos eran detritívoros y $11,11 \%$ de herbívoros y para el segundo sistema estaba representando con el $56 \%$ de organismos detritívoros, $35 \%$ de herbívoros y $9 \%$ de depredadores. Esto confirma con los resultados de esta investigación, ya que, de la población total en ambas épocas, el $86 \%$ está constituido por los detritívoros. Esto es gracias por la cantidad de biomasa vegetal que aporta los árboles permitiendo fuentes de alimentos para estos organismos y acondicionando su nicho ecológico.

\section{CONCLUSION}

Los arreglos agroforestales estudiados no difieren entre sí en la abundancia y riqueza de los organismos encontrados. Se encontraron 26 familias en la época lluviosa y 30 familias en la época seca. El número de individuos recolectados en la época lluviosa fue de 391 y en la época seca 324. En la época lluviosa el sistema que presentó mayor abundancia fue CMIEA con 163 individuos de igual manera en la época seca con 129 individuos. La mayor cantidad de macroinvertebrados se localizaron en los primero $10 \mathrm{~cm}$ del suelo predominando organismos detritívoros como las Philoscidae y organismo del orden haplotaxida. De acuerdo con el índice de Shannon el sistema que presentó mayor riqueza fue sistema agroforestal CA (2.73) en la época seca seguido por el sistema CMIEA (2.62) en la época lluviosa. 


\section{REFERENCIAS}

Anderson, J. M. e Ingram, J. S. I. (1993). Tropical soil biology and fertility: a handbook of methods. 2nd ed. Wallingford. CABI. p. 240.

Adrews K, L. y Caballero, R (1990). Guía para el estudio de órdenes y familias de insectos de Centroamérica. Escuela agrícola panamericana, el Zamorano, Honduras, p 180.

Blair, J; Bohlen J \& Freckman D. (1996). Soil invertebrates as indicators of soil quality. P 273-291

Coto-Alfaro, D. (1998). Descripción taxonómica de plagas de importancia agrícola del Orden Lepidoptera. Manejo Integrado de Plagas., (10), 72-92.

Coral, D.M. \& Bonilla, C.R. (1998) Impacto de las prácticas agrícolas sobre la macrofauna del suelo en la cuenca alta del lago Guamues, Pasto, Colombia. Acta agronómica. 18 (3-4):55.

Chávez Suárez, L., Labrada, Y., Rodríguez García, I., Álvarez Fonseca, A., Bruqueta Yero, D., \& Licea Castro, L. (2018). Caracterización de la macrofauna edáfica en un pastizal de la provincia Granma. Centro Agrícola, 45(4), 43-48.

García, Y.;Ramírez, w. y Sánchez, S. (2014). Efecto de diferentes usos de la tierra en la composición y la abundancia de la macrofuna edáfca, en la provincia Matanzas. Pastos y Forrajes, 37 (3): 313-321.

Govaerts, B.; Sayre, K. D. \& Deckers, J. (2006). A minimum data set for soil quality assessment of wheat and maize cropping in the highlands of Mexico. Soil \& Tillage Research. 87: 163.

King, A. B., \& Saunders, J. L. (1984). The invertebrate pests of annual food crops in Central America: A guide to their recognition and control. Bib. Orton IICA/CATIE.

Lavelle, P.; Decaëns, T.; Aubert, M.; Barot, S.; Blouin, M.; Bureau; et al. (2006). Soil invertebrates and ecosystem services. European Journal of Soil Biology. 42:3.

LOK, S. (2005). Determinación y selección de indicadores del sistema suelo-pasto en pastizales dedicados a la producción de ganado vacuno. Tesis presenta en opción al grado científco de Doctor en Ciencias Agrícolas. Instituto de Ciencia Animal, La Habana, Cuba, 98 p.

Moreira, F., E. J. Huising y D. E. Bignell. (2012). Manual de biología de suelos tropicales. Muestreo y caracterización de la biodiversidad bajo suelo. Instituto Nacional de Ecología, México. 337 pp.

MAAS, J:L (1998). Compendiumm of Strawberry Deseases US Department of Agriculture Beltsville, Maryland. Second Edition. P 98.

Pardo, L.; Lelez, C.; Sevilla, F.; Madrid, O. (2006). Abundancia y biomasa de macroinvertebrados edáficos en la temporada de lluvias en tres usos de tierra en los Andes, Colombianos. s.n.p. 12p.

Primavesi, A. (1984). Manejo ecologico do solo; a agricultura em regioes tropicais.

Rendon, S.; Artunduag, F.; Ramirez, R.; Quiroz, J.; Leiva, E. (2011). Los macroinvertebrados como indicadores de la calidad del suelo en cultivo de mora, pasto y aguacate. Rev. Fac. Agr. Mendellin. 64(1):5791-5802 
Sánchez, S. (2007). Acumulación y descomposición de la hojarasca en un pastizal de Panicum maximum Jacq. y en un sistema silvopastoril de P. maximum y Leucaena leucocephala (Lam.) de Wit. Tesis presentada en opción al grado científico de Doctor en Ciencias Agrícolas. Instituto de Ciencia Animal. La Habana, Cuba.135 p.

Sánchez, S. y Reyes, F. (2003). Estudio de la macrofauna edáfica en una asociación de Morus alba y leguminosas arbóreas. Pastos y Forrajes. 26:315

Serna, F. J.; Vergara, E. V. 2001. Claves para la identificación de subfamilias y géneros de hormigas de Antioquia y Chocó, Colombia. Revista del Instituto de Ciencias Naturales y Ecología. 7:5-41. 


\section{APÉNDICE}

Tabla 3: Abundancia y diversidad de macrofauna edáfica en sistemas agroforestales en época seca y lluviosa.

\begin{tabular}{|c|c|c|c|c|c|c|c|c|c|}
\hline \multirow[b]{2}{*}{ Clase } & \multirow[b]{2}{*}{ Familia } & \multicolumn{3}{|c|}{ Época lluviosa } & \multicolumn{5}{|c|}{ Época seca } \\
\hline & & CMIEA & CIE & $\mathbf{C A}$ & Total/Individuo & CMIEA & CIE & $\mathbf{C A}$ & Total/ individuo \\
\hline \multirow[t]{25}{*}{ Insecta } & Formicidae & 20 & 1 & 4 & 25 & 11 & 2 & 10 & 23 \\
\hline & Chrysomelidae & 2 & & & 2 & & & & \\
\hline & Scarabaeidae & 5 & 2 & 10 & 17 & 3 & 1 & 3 & 7 \\
\hline & Lampyridae & & & 4 & 4 & 1 & 2 & & 3 \\
\hline & Elateridae & 1 & 1 & 1 & 3 & & 1 & & 1 \\
\hline & Curculionidae & & & & & & 1 & & 1 \\
\hline & Tenebrionidae & & & & & & 1 & 1 & 2 \\
\hline & Coccinellidae & & & & & & & 1 & 1 \\
\hline & Polyphagidae & 1 & & & 1 & & & & \\
\hline & Blattidae & & & & & 2 & & & 2 \\
\hline & Blattellidae & & & & & & & 4 & 4 \\
\hline & Polyphagidae & & & & & & 1 & 1 & 2 \\
\hline & Blaberidae & & & 1 & 1 & 1 & 2 & & 3 \\
\hline & Cydnidae & 1 & & 1 & 2 & & & 1 & 1 \\
\hline & Reduviidae & & & & & 1 & & & $\mathbf{1}$ \\
\hline & Coreidae & & & & & 1 & & & 1 \\
\hline & Nabidae & 1 & & & 1 & 2 & 1 & & 3 \\
\hline & Stratiomydae & 2 & & & 2 & 6 & & & 6 \\
\hline & Gryllidae & 2 & 1 & 1 & 4 & 1 & & 1 & 2 \\
\hline & Tetrigidae & & 1 & 1 & 2 & & & & \\
\hline & Tettigoniidae & & & 1 & 1 & 1 & & 1 & 2 \\
\hline & Anisolabididae & & & 1 & 1 & & & & \\
\hline & Labiduridae & & & & & 1 & & & 1 \\
\hline & Geometridae & & & & & & & 1 & $\mathbf{1}$ \\
\hline & Erebidae & & & & & & & 1 & 1 \\
\hline \multirow[t]{9}{*}{ Arachnida } & Hahniidae & 4 & 1 & & 5 & & & & \\
\hline & Lycosidae & 1 & & & 1 & 2 & & & 2 \\
\hline & Salticidae & & & & & 1 & & 1 & 2 \\
\hline & Linyphiidae & & & & & & & 1 & $\mathbf{1}$ \\
\hline & Araneidae & & & & & & 1 & & $\mathbf{1}$ \\
\hline & Oxiopidae & & 1 & & 1 & & & & \\
\hline & Phalangiidae & & & & & 1 & & 1 & 2 \\
\hline & Tetranychidae & 1 & & & 1 & & & & \\
\hline & Chernetidae & 1 & & & 1 & & & & \\
\hline Melacostraca & Philosciidae & 37 & 29 & 26 & 92 & 8 & 5 & 11 & 24 \\
\hline Diplopoda & Paradoxosomatidae & 1 & & & 1 & & & & \\
\hline
\end{tabular}




\begin{tabular}{llcccccccc} 
& Julidae & 1 & & 1 & $\mathbf{2}$ & 1 & & $\mathbf{1}$ \\
Chilopoda & Scolopendridae & 3 & & 1 & $\mathbf{4}$ & & 1 & 4 & $\mathbf{5}$ \\
& Geophilidae & 2 & 1 & 5 & $\mathbf{8}$ & & 2 & & $\mathbf{2}$ \\
Gastropoda & Veronicellidae & & & 1 & $\mathbf{1}$ & & & & \\
Oligochaeta & & 77 & 73 & 58 & $\mathbf{2 0 8}$ & 85 & 83 & 48 & $\mathbf{1 0 8}$ \\
\hline & & $\mathbf{1 6 3}$ & $\mathbf{1 1 1}$ & $\mathbf{1 1 7}$ & $\mathbf{3 9 1}$ & $\mathbf{1 2 9}$ & $\mathbf{1 0 4}$ & $\mathbf{9 1}$ & $\mathbf{3 2 4}$ \\
\hline
\end{tabular}

CMIEA= Café, musáceas, Inga, Erythrina y árboles; CIEA= Café, Inga y Erythrina y árboles; CA= Café y Árboles.

\section{SEMBLANZA DE LOS AUTORES}

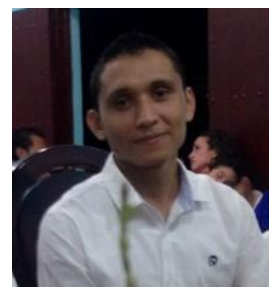

Conrado R. Quiroz-Medina: Obtuvo el grado de Ingeniero en Agroecología Tropical en la Universidad Nacional Autónoma de Nicaragua - León, donde actualmente es Profesor Encargado de cátedra e investigador de la Escuela de Ciencias Agrarias y Veterinaria. Ha trabajado en proyectos de investigación en conjunto con organizaciones independiente e instituciones estatales. Actualmente trabaja en línea de investigación vinculada en la identificación de insectos plagas, control biológico, macrofauna edáfica y acuática. Es parte del equipo técnico como diseñador, maquetador y revisor de la Revista Iberoamericana de Bioeconomía y Cambio Climática.



Juan Duley Castellón: Obtuvo el grado de Ingeniero Agrónomo en la Universidad Nacional Agraria, actualmente es profesor asistente de la UNAN-León, investigador y líder de proyectos Gestión de los Recursos Hídricos Mayor Resiliencia Frente a la variabilidad Climática con FONTAGRO, coordinador del proyecto Musáceas y representante por Nicaragua de La Red de Musáceas de América Latina y el Caribe Musalac. Desarrollo sus estudios de maestría en Agricultura Ecológica en el Centro Internacional de Investigaciones y Enseñanzas (CATIE). Ha desarrollado proyectos de colaboración Regionales en Bananos en cafetales con Bioversity International y el IDIAF de La Republica Dominicana. Coordinadro de la Unidad de Investigación Eco intensificación del CICAEA.

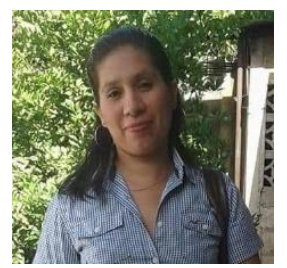

Noelia E. Cea-Navas: Obtuvo el grado de Licenciada en Estadística en la Universidad Nacional Autónoma de Nicaragua - León, donde actualmente es profesor adjunto e investigador. Ha trabajado en proyectos de investigación en conjunto con el Banco de Semillas Forestales (CMG\&BSF del INAFOR), en la validación de material genético de diferentes arboles forestales. Trabaja en proyectos en el sector acuícola como la identificación de ectos y endo parásitos en peces estuarios, en identificación de especies de bivalvos y eficiencia nutricional de dietas balanceadas para el cultivo de pargo, también trabaja en proyectos como la degradación de la molécula química plaguicida en tres sustratos. Actualmente trabaja en líneas de investigación vinculadas con el sector.

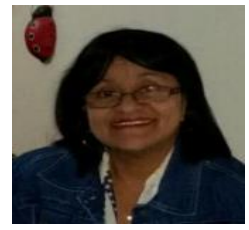

Mirna S. Ortiz. Obtuvo el grado de Licenciada en Biología en la Universidad Nacional Autónoma de Nicaragua - León, docente retirada de la UNAN, León, trabaja en investigaciones independiente en el área de Entomología.

Carlos A. Zuniga-Gonzalez: Obtuvo el grado de Economista Agrícola en la Universidad Nacional Autónoma de Nicaragua, Managua, actualmente es profesor titular de la UNAN-León, investigador

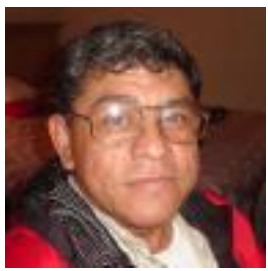
y Director del Centro de Investigación en Ciencias Agrarias y Economía Aplicada. Desarrolló sus estudios de maestría en la Universidad de Barcelona España y su doctorado en el American World University, EU sede Guatemala. Ha desarrollado estancias de investigación en universidades extranjeras con académicos reconocidos dentro de su campo de su especialidad. Es coordinador de la Red REBICAMCLI especializándose en temas de la Bioeconomía, Análisis de productividad, Cambio Climático. Y es editor en jefe de la revista Iberoamericana de Bioeconomía y Cambio Climático de la UNAN- León. 